\title{
A three-dimensional deformable model for segmentation of human prostate from ultrasound images
}

\author{
Amir Ghaneia) \\ Department of Radiology, Henry Ford Health System, Detroit, Michigan 48202 \\ and University of Michigan, Ann Arbor, Michigan 48105 \\ Hamid Soltanian-Zadeh \\ Department of Radiology, Henry Ford Health System, Detroit, Michigan 48202 \\ and University of Tehran, Tehran, 14399, Iran \\ Alexander Ratkewicz and Fang-Fang Yin \\ Department of Radiation Oncology, Henry Ford Health System, Detroit, Michigan 48202
}

(Received 14 September 2000; accepted for publication 16 May 2001)

\begin{abstract}
Segmentation of human prostate from ultrasound (US) images is a crucial step in radiation therapy, especially in real-time planning for US image-guided prostate seed implant. This step is critical to determine the radioactive seed placement and to ensure the adequate dose coverage of prostate. However, due to the low contrast of prostate and very low signal-to-noise ratio in US images, this task remains as an obstacle. The manual segmentation of this object is time consuming and highly subjective. In this work, we have proposed a three-dimensional (3D) deformable surface model for automatic segmentation of prostate. The model has a discrete structure made from a set of vertices in the 3D space that form triangle facets. The model converges from an initial shape to its equilibrium iteratively, by a weighted sum of the internal and external forces. Internal forces are based on the local curvature of the surface and external forces are extracted from the volumetric image data by applying an appropriate edge filter. We have also developed a method for initialization of the model from a few initial contours that are drawn on different slices. During the deformation, a resampling procedure is used to maintain the resolution of the model. The entire model is applied in a multiscale scheme, which increases the robustness and speed, and guarantees a better convergence. The model is tested on real clinical data and initial results are very promising. (c) 2001 American Association of Physicists in Medicine. [DOI: 10.1118/1.1388221]
\end{abstract}

Key words: segmentation, deformable model, prostate, ultrasound, radiation therapy

\section{INTRODUCTION}

Prostate boundaries form the basis of many diagnosis/ treatment procedures for prostate diseases. Prostate implant is a common procedure in radiation therapy, which requires outlining the prostate boundaries. However, there is a large intraoperator variation in manual contouring, and it is not reproducible. Also the time needed for contouring prostate is not desirable for a real-time prostate implant procedure. The goal of this research was to develop a technique to semiautomatically and consistently segment prostate in ultrasound images, and to improve the efficiency of a real-time prostate implant procedure.

There have been a number of works so far on automatic segmentation of prostate from ultrasound (US) images. Pathak et al. ${ }^{1}$ used an edge-based technique for outlining the prostate and statistically showed that automatic or semiautomatic segmentation of the prostate indeed leads to a better consistency of the results. The segmentation technique of Ladak et $a l^{2}$ is based on a deformable model that changes shape to fit the boundary of an object. They used anatomic data and cubic interpolation to interpolate between points. In their model, gradient direction information is used during deformation to push the model toward the boundaries. However, the success of their approach is dependent on the care- ful initialization of the contour, which requires the user to select points on the prostate boundary.

Knoll et al. ${ }^{3-5}$ have considered the problem of deformable contour initialization and modeling for segmentation of the human prostate in medical images. They proposed a technique for elastic deformation restriction to particular object shapes of closed planar curve using localized multiscale contour parametrization based on the one-dimensional dyadic wavelet transform (WT). For this purpose, they define internal curve deformation forces as a result of multiscale parametrical contour analysis. Their contour deformation method is integrated into a coarse-to-fine segmentation frame based on a multiscale image edge representation using the local modulus maxima of the dyadic WT. The form restricted contour deformation and its initialization by template matching are performed in a coarse to fine segmentation process based on a multiscale image edge representation containing the important edges of the image at various scales.

Chen et al. ${ }^{6,7}$ have presented an algorithm to reconstruct the three-dimensional (3D)-shading image of the prostate from a series of ultrasound cross-sectional images of the organ. In their proposed method, first the image is smoothed by low pass filtering. The center of the prostate is estimated and then a search process in radial directions from the estimated 
center is performed to locate the prostate boundary by matched filtering. Then they use edge linking to obtain the two-dimensional (2D) contour. The Hermite-surface approach is used to interpolate the spatial data to reconstruct the $3 \mathrm{D}$ object's surface from a series of $2 \mathrm{D}$ contours.

Richard et al. ${ }^{8}$ have segmented 2D images of the prostate gland into prostate and nonprostate regions for forming a 3D image of the prostate from a set of parallel 2D images. The texture-based segmentation method they presented is a pixel classifier based on four texture energy measures associated with each pixel in the image. A clustering procedure is used to label each pixel in the image with the label of its most probable class.

Prater et al. ${ }^{9}$ described a method for segmenting transrectal ultrasound images of the prostate using feed-forward neural networks. They presented three neural network architectures for this purpose. Each of these networks was trained using a small portion of a training image segmented by an expert sonographer.

Englmeier ${ }^{10}$ suggested a method that with the aid of computer enables the user to obtain data on volume and weight by segmentation and integration of pictures of sections of the gland. The authors described the methods used to extract the required data from the ultrasonic images and evaluate the results obtained.

In our approach, we use a 3D discrete deformable surface for accurate outlining of prostate. After acquiring the 2D ultrasound images, we use bilinear interpolation to create a $3 \mathrm{D}$ volumetric image. The operator draws a few initial contours on some (typically $40 \%-70 \%$ ) of the slices. These initial contours, which are outlined as polygons, do not have to be accurate and can have as low as four to five points. The model creates a closed initial surface from the contours drawn. Starting from the initial surface, the model deforms by movement of its vertices under defined force termsinternal and external forces. Calculated from the local surface curvature, internal forces try to maximize the model smoothness, while external forces pull the model toward the prostate boundaries. We have applied the proposed model to real clinical cases and compared the results with manual outlining. In the following, we first give a brief description of the model and then we discuss the results.

\section{METHODS AND MATERIALS}

\section{A. Model structure}

This section briefly describes the model geometry. The reader is referred to Refs. 11 and 12 for more details. The model structure consists of a set of vertices and edges that form a closed surface defined by connected triangle facets (see Fig. 1). The position of vertex $i, V_{i}$, in the Cartesian space is represented by vector $\mathbf{p}_{i}$. Each vertex, like $i$, is directly connected to three or more vertices by edges. The $k$ th neighboring vertex of $V_{i}$ is shown by $v(i, k) \quad(0 \leqslant k$ $\leqslant M_{i}-1$, where $M_{i}$ is the number of the neighboring vertices for $\left.V_{i}\right)$. Looking to $V_{i}$ from outside of the volume per-

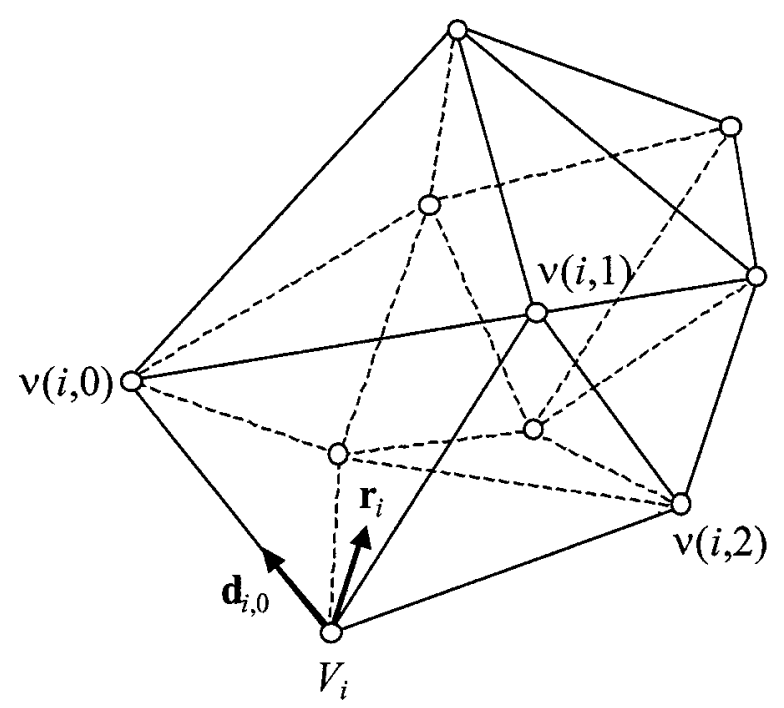

FIG. 1. A simple example of the model structure with ten vertices and five patches. The relative indices of some of the neighbors of $V_{i}$ have been shown. $\mathbf{r}_{i}$ is the unit radial (normal) vector at vertex $i$ and $\mathbf{d}_{i, 0}$ is the unit vector along the edge between $V_{i}$ and $v(i, 0)$.

pendicular to the surface, the index $k$ in $v(i, k)$ increases in the clockwise direction. The edge between $i$ and $v(i, k)$ is shown by $\mathbf{D}_{i, k}$ :

$$
\mathbf{D}_{i, k}=\mathbf{p}_{v(i, k)}-\mathbf{p}_{i} \text {. }
$$

We use $\mathbf{d}_{i, k}$ to show the unit vector along $\mathbf{D}_{i, k}$ and $\mathbf{r}_{i}$ to show the unit vector normal to the model surface at $V_{i}$.

\section{B. Model deformation}

The model deforms under the influence of the internal and external forces. The external forces are extracted from the image features. In this work, external forces are defined to make the model converge to the object boundaries. The internal forces maintain the smoothness of the model by minimizing the surface curvature as described in Sec. II B 1.

\section{Internal forces}

The internal forces are based on the local curvature of the surface. In this work, we use a least-squares error estimation to the Dupin indicatrix for estimating the curvature and the surface normal. ${ }^{13}$ The method is robust and relatively insensitive to the model resolution, number of points used for estimation, and choice of the coordinate system. After calculating the principal curvatures (maximum and minimum curvatures) for each vertex, $\kappa_{1}$ and $\kappa_{2}$, we consider the mean curvature, $C_{i}$, as the numerical value for the curvature at that vertex:

$$
C_{i}=\left(\kappa_{1}+\kappa_{2}\right) / 2 \text {. }
$$

Internal force at vertex $i, \mathbf{f}_{\mathrm{in}, i}$, is computed from the local curvature value, $C_{i}$, and the curvatures of the neighboring vertices, $C_{v(i, k)}$, as

$$
\mathbf{f}_{\mathrm{in}, i}=\left\{C_{i}-\frac{1}{M_{i}} \sum_{k=0}^{M_{i}-1} C_{v(i, k)}\right\} \mathbf{r}_{i} .
$$




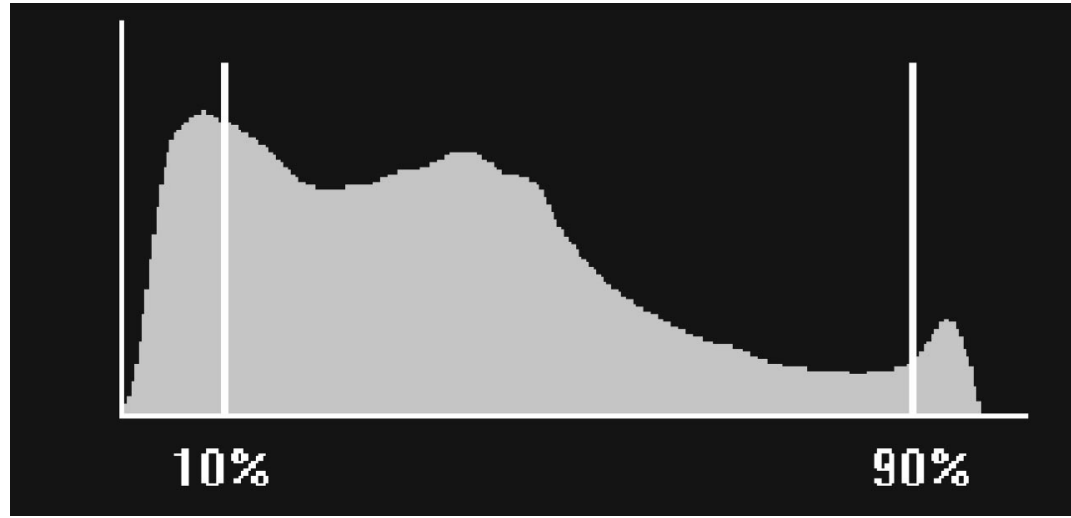

FIG. 2. The histogram of a typical prostate ultrasound image. The pixels that are too bright (with gray levels larger than $90 \%$ of the maximum image gray level) or too dark (with gray levels smaller than $10 \%$ of the maximum image gray level) are not considered for calculation of the image energies, $E_{\mathrm{im}}$.

The above-given definition sets the direction of $\mathbf{f}_{\mathrm{in}, i}$ along the radial vector. When $\mathbf{f}_{\text {in }}$ is the only acting force, the abovementioned internal forces push the initial shape toward a maximally smooth and closed surface, which has a general spherical shape. In practical situations, there are also external forces acting on the model, and the final shape depends on the relative weight of the two forces and will also be affected by the image data.

\section{External forces}

External forces push the model toward the minimum of an external energy, which is defined from the volume data using an appropriate operator. We use a step expansion matching and restoration (SEF) filter which has been introduced by Rao et al. ${ }^{14}$ It is an edge detector that uses expansion matching and restoration. It is optimal in the sense of a special figure of merit named discriminative signal-to-noise ratio. ${ }^{14}$ Assuming the noise in the image can be modeled with white noise and the edge model is a step function, the impulse response of SEF filter in one dimension is

$$
h(x)=\operatorname{sgn}(x) e^{-2|x| / \sigma},
$$

where $\sigma^{2}$ is the variance of the white noise and $\operatorname{sgn}(x)$ is the sign function. Note that the noise in the US images includes white noise and speckle noise. It is possible to use other filters that are specifically designed for US images. ${ }^{15}$

The above-mentioned filter is applied to the volumetric image data in three perpendicular directions and the sum of the results is used as the image energy. The gradient of this image energy at each point defines the local external force. The force applied to each vertex is the radial component of the local external force. Hence, we will have

$$
\mathbf{f}_{\mathrm{ex}, i}=-\left(\nabla E_{\mathrm{im}} \cdot \mathbf{r}_{i}\right) \mathbf{r}_{i},
$$

where $E_{\mathrm{im}}$ is the image energy defined by

$$
E_{\mathrm{im}}=|h(x) * I|+|h(y) * I|+|h(z) * I|+\nabla \delta,
$$

where $\nabla \delta$ is the gradient of the local standard deviation of the image data, measured in a window centered at the point of interest, $I$ represents the volumetric image data, and $*$ is the convolution operator.

Effects of the strong edge at the interface of ultrasound probe and body tissue, and artifacts generated by destructive and constructive addition of the ultrasound waves create some very strong gradients in the image that can mislead the model. To remedy this problem, we ignore the pixels that are too bright or too dark in calculation of $E_{\mathrm{im}}$. We have experimentally found that ignoring the first $10 \%$ and last $10 \%$ of the histogram will considerably reduces this undesired effect. Figure 2 shows the histogram for a typical US prostate image and the cutoff points.

\section{Deformation process}

The deformation process is a result of internal and external forces acting on the model. The total force acting on each vertex is a weighted sum of $\mathbf{f}_{\mathrm{in}}$ and $\mathbf{f}_{\mathrm{ex}}$ and a damping force,

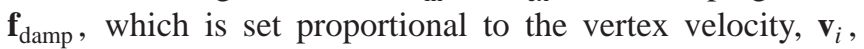
and used to make the model more stable:

$$
\mathbf{f}_{\text {damp }, i}=K_{\text {damp }} \mathbf{v}_{i},
$$

where $K_{\text {damp }}$ is a negative constant. The surface deformation is computed in discrete positions in time as follows:

$$
\begin{aligned}
& \mathbf{p}_{i}(t+\Delta t)=\mathbf{p}_{i}(t)+\mathbf{v}_{i}(t) \Delta t, \\
& \mathbf{v}_{i}(t+\Delta t)=\mathbf{v}_{i}(t)+\mathbf{a}_{i}(t) \Delta t, \\
& \mathbf{a}_{i}(t+\Delta t)=\mathbf{f}_{i}(t+\Delta t) / m_{i}, \\
& \mathbf{f}_{i}=w_{\mathrm{ex}} \mathbf{f}_{\mathrm{ex}, i}+w_{\mathrm{in}} \mathbf{f}_{\mathrm{in}, i}+w_{\mathrm{damp}} \mathbf{f}_{\mathrm{damp}, i},
\end{aligned}
$$

where $\mathbf{a}_{i}, \mathbf{v}_{i}$, and $m_{i}$ are vertex acceleration, velocity, and assigned mass, respectively. $\Delta t$ is the time step and its value can influence the model convergence. We have used $\Delta t$ $=1 \mathrm{~s}$ in each iteration. A larger value might jeopardize the convergence and too small values slow down the model.

The deformation process starts from an initial surface. Vertices will move according to Eqs. (8)-(11) until the model reaches equilibrium in which the total force acting on each vertex is zero. In practice, we use a threshold on the model velocity as a stopping criterion. In this method, it is assumed that convergence is achieved when the model movements are very small during several consecutive iterations.

For the model initialization, most of the authors have used a fixed shape. We have developed a specific method that gives the user flexibility in defining the initial shape. The model creates a closed surface from a stack of 2D contours 
TABLE I. Comparison between the model results and manual outlining for ten studies. Pixelwise OR, AND, and XOR operators are used to measure the similarity and difference between two areas (manual and model). Similarity is defined by the ratio of the common area to the total area, and the difference as the ratio of noncommon area to the total area. The average distances of manual and model contours are shown in the last column.

\begin{tabular}{ccccccc}
\hline \hline $\begin{array}{c}\text { Prostate } \\
\text { study }\end{array}$ & $\begin{array}{c}\text { Average } \\
\text { OR } \\
\text { (pixels) }\end{array}$ & $\begin{array}{c}\text { Average } \\
\text { AND } \\
\text { (pixels) }\end{array}$ & $\begin{array}{c}\text { Average } \\
\text { XOR } \\
\text { (pixels) }\end{array}$ & $\begin{array}{c}\text { Average } \\
\text { similarity } \\
(\%)\end{array}$ & $\begin{array}{c}\text { Average } \\
\text { difference } \\
(\%)\end{array}$ & $\begin{array}{c}\text { Average } \\
\text { distance } \\
\text { (pixels) }\end{array}$ \\
\hline 1 & 4903 & 4176 & 727 & 85.00 & 15.00 & 2.74 \\
2 & 4746 & 4086 & 660 & 85.71 & 14.29 & 2.49 \\
3 & 7885 & 6788 & 1096 & 85.67 & 14.33 & 2.88 \\
4 & 5897 & 5251 & 645 & 89.43 & 10.57 & 2.25 \\
5 & 5011 & 4533 & 478 & 90.46 & 9.54 & 1.84 \\
6 & 6123 & 5347 & 775 & 90.43 & 9.57 & 2.32 \\
7 & 5515 & 4886 & 628 & 88.69 & 11.31 & 2.30 \\
8 & 4882 & 4242 & 639 & 89.60 & 10.40 & 2.00 \\
9 & 6300 & 5671 & 729 & 91.67 & 8.33 & 2.64 \\
10 & 12004 & 10777 & 1227 & 89.13 & 10.87 & 2.92 \\
& Average of averages & & 88.58 & 11.42 & 2.44 \\
\hline \hline
\end{tabular}

that have been drawn on parallel cross sections. On possible approach for achieving this goal is to apply a generic triangulation method to the set of initial vertices. In our model, the initial shape will be produced in two steps. In the first step, appropriate vertices of the consecutive polygons are connected to each other in a specific order. In the second step, the first and the last polygons are broken into triangle facets, as explained in detail in Ref. 11.

\section{Prostate studies}

We acquired ultrasound prostate image sets of ten patients. We used a dual-focus multiplane $7.5 \mathrm{MHz}$ transrectal US probe. Images were acquired in a pre-planned clinical condition. The US device was directly connected to a computer and images stored digitally. In each study, the images are $5 \mathrm{~mm}$ apart, and have a pixel size of $0.36 \times 0.36 \mathrm{~mm}$, and the matrix size of $256 \times 256(\mathrm{FOV}=9 \times 9 \mathrm{~cm})$. The initial contours are drawn on $40 \%-70 \%$ of the slices of each study, and six to eight vertices used for each 2D contour. Then the contours are connected to form a 3D initial shape for the model. The running time is approximately $30 \mathrm{~s}$ on a SUN Ultra 20. The same studies are segmented carefully by a radiologist and the contours are compared using the methods in Sec. II D.

\section{Evaluation methods}

We use a similarity measure, which is based on Kappa statistics to compare the model results with the manual segmentation. For measuring the similarity value, let $A_{1}$ and $A_{2}$ be the areas (in pixels) obtained by the manual and automatic methods, respectively. The relative amount of agreement (similarity value) and disagreement between the model and manual segmentation are shown by $R_{c}$ and $R_{n}$, respectively, and for each slice they are computed as

$$
R_{c}=\frac{A_{1} \text { AND } A_{2}}{A_{1} \text { OR } A_{2}}
$$

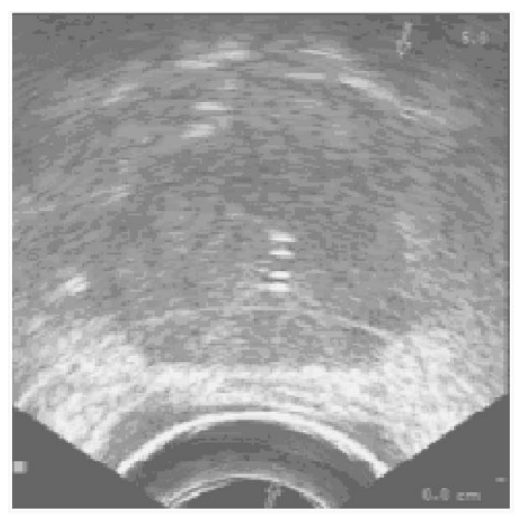

(a)

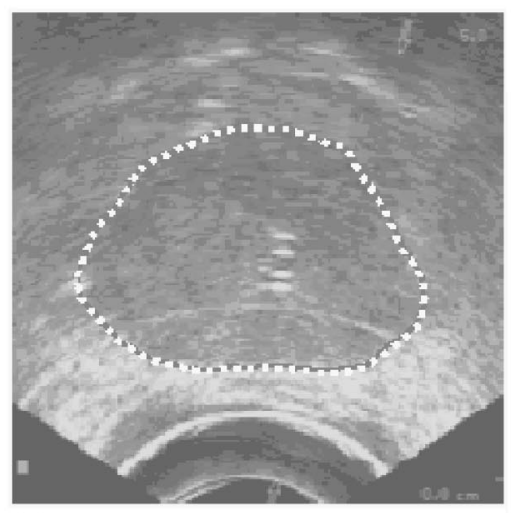

(c)

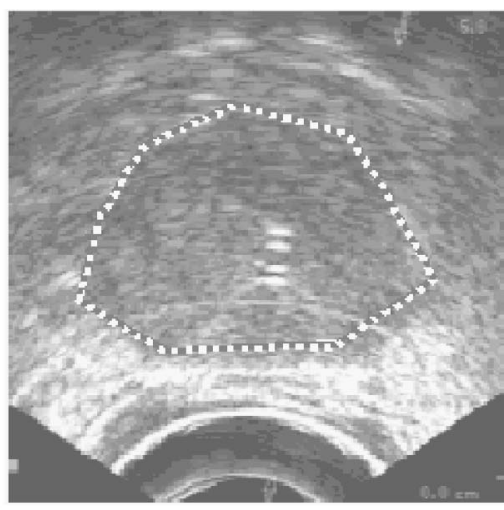

(b)

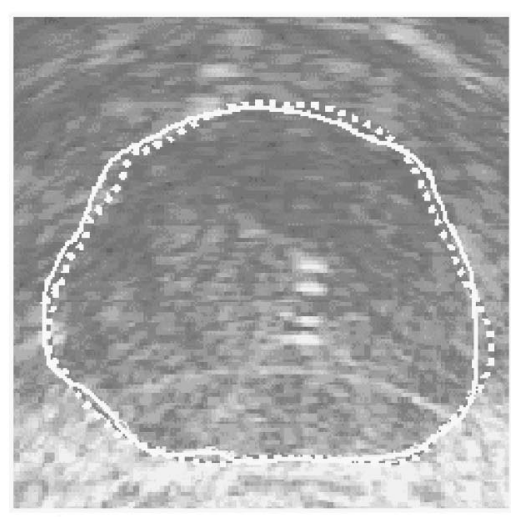

(d)
FIG. 3. (a) A slice of the ultrasound prostate image. (b) The prostates image with the initial contour overlaid (dash lines). (c) The prostate image with the final contour generated by the model. (d) The prostate image with both the manual contour (solid lines) and the model contour (dashed lines). Note the good agreement between the model and manual contours. 


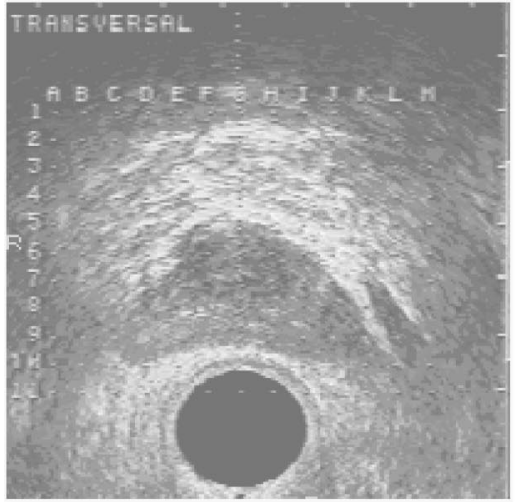

(a)

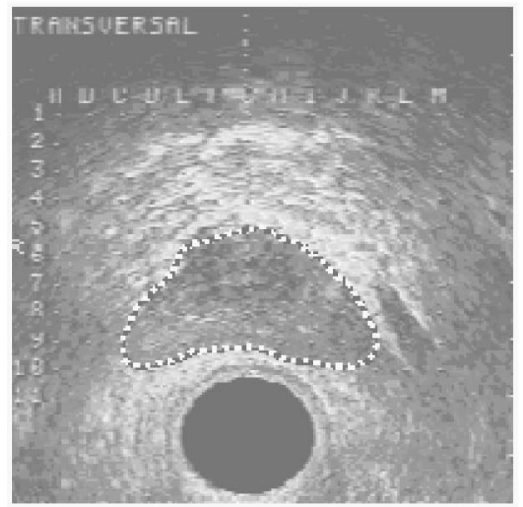

(c)

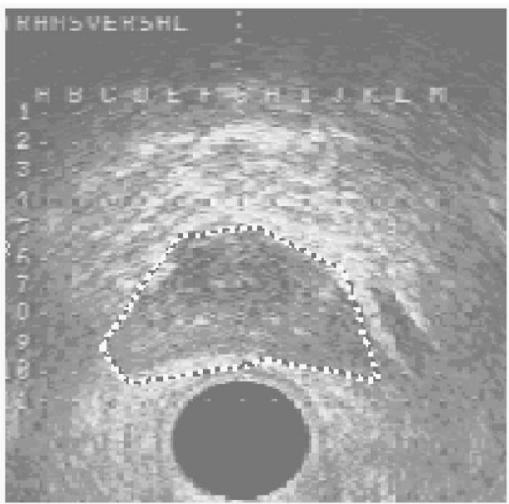

(b)

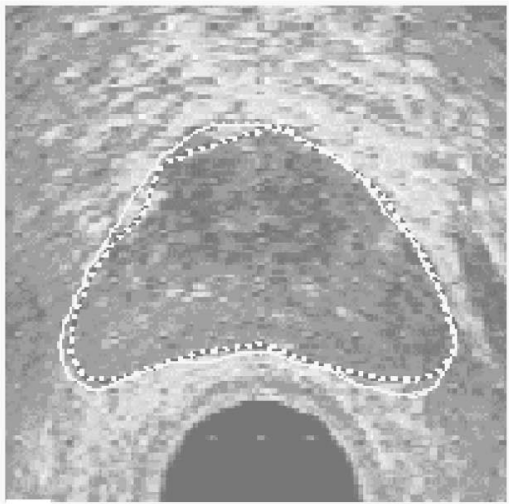

(d)

FIG. 4. (a) A slice of the ultrasound prostate image. (b) The prostate image with the initial contour overlaid (dashed lines). (c) The prostate image with the final contour generated by the model. (d) The prostate image (using a larger magnification) with both the manual contour (solid lines) and the model contour (dashed lines). Note that the model has successfully closed the boundaries at the bottom left and right of the prostate where there are no clear edges. Also, the strong edge at the interface between the ultrasound probe and tissue (dark circle at the bottom of the image) has not misled the model.

$$
R_{n}=\frac{A_{1} \text { OR } A_{2}-A_{1} \text { AND } A_{2}}{A_{1} \text { OR } A_{2}},
$$

where $R_{c}$ and $R_{n}$ are the ratio of the common pixels (pixels that marked as prostate by both methods), and noncommon pixels (pixels that are marked only by one method) to the total area, respectively. The common pixels are obtained by pixelwise AND operator and total area by pixelwise OR operator. A similarity value between 0.7 and 1.0 represents an excellent agreement while a similarity between 0.4 and 0.7 represents a good agreement.

Another measure we use to evaluate the results is the average distance between the model and the manual contours. This is calculated by finding the nearest pixel on the other contour for each pixel on the model contour and getting the average of Euclidean distance of the pixels along the contour.

\section{EXPERIMENTAL RESULTS}

We applied the proposed deformable model to ten ultrasound image sets described in Sec. II C to segment the prostate. A physician also segmented the prostate by drawing its boundaries on individual slices using a mouse. Then we compared manual segmentation results with the model using the methods explained in Sec. II D.

The summary of the performance measures are presented in Table I. Each row shows the average of the values over all the slices of the corresponding patient. It can be seen from Table I that the average similarity value is $0.89(89 \%)$ and there exists an excellent agreement between the results obtained by the two methods. We also measured the average distance of the model and manual contours, which is about 2.5 pixels as reported in Table I.

Figure 3 shows the result on a slice generated by the model. The initial and final model contours are shown using dashed line. The manual contour is shown using solid line. As can be seen there is good agreement between the model and the manual contour.

In Fig. 4, a slice near the end of prostate is shown. In this example, the model successfully closed the boundary at the bottom left and right where there is no clear edge. The strong edges created at the interface of ultrasound probe and tissue has not misled the model. In Fig. 5, a beginning slice of prostate is shown along with the manual result. Parts of the difference between the manual and model contour on this image is due to the smooth closing of the model at the end slices versus the abrupt end in manual segmentation.

\section{DISCUSSION}

It can be seen that there is excellent agreement between the model and radiologist. We observed that radiologists tend to draw the contours rather large in areas that do not have a clear boundary. Part of the disagreement between the model and manual contours can be attributed to this fact. Also, disagreement was largest on the beginning and end slices due to smooth closing of the 3D model versus abrupt start and end that a radiologist applies. 


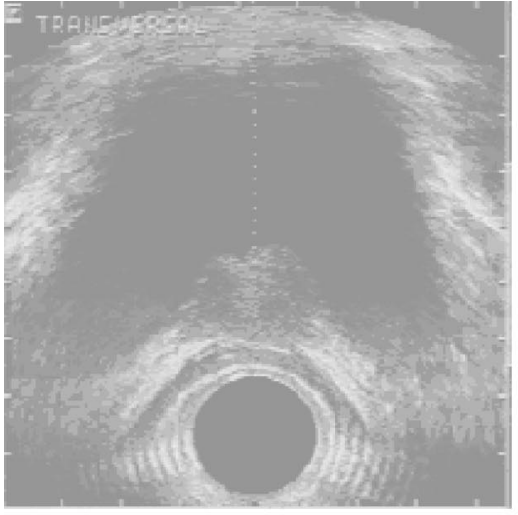

(a)

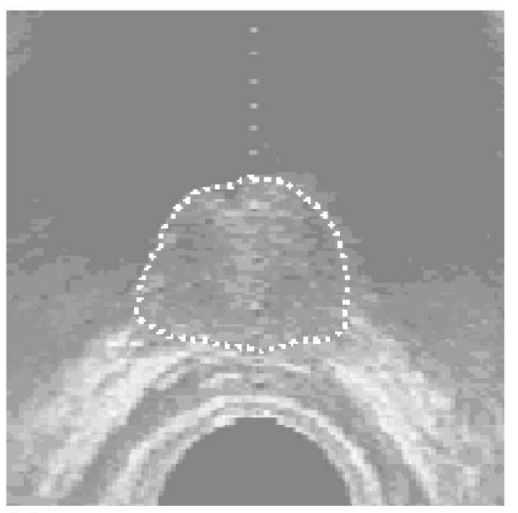

(c)

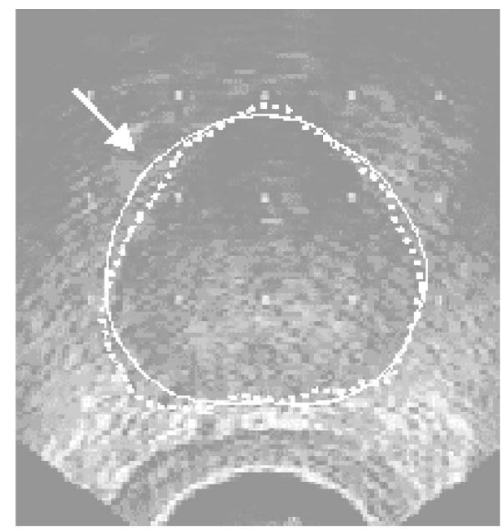

(a)

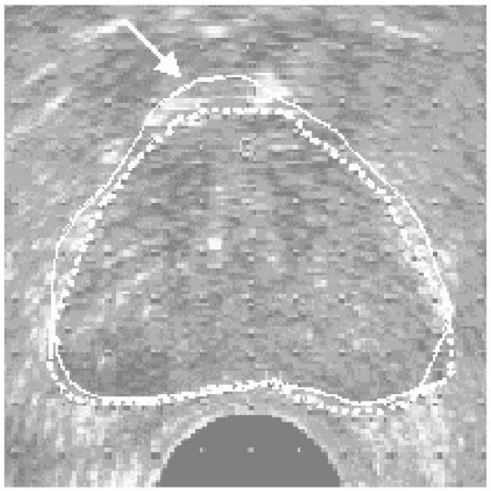

(c)

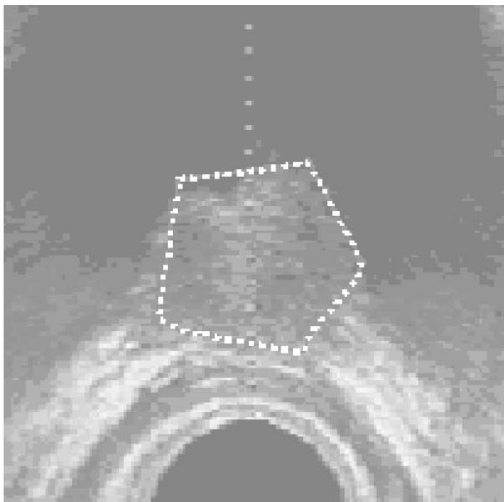

(b)

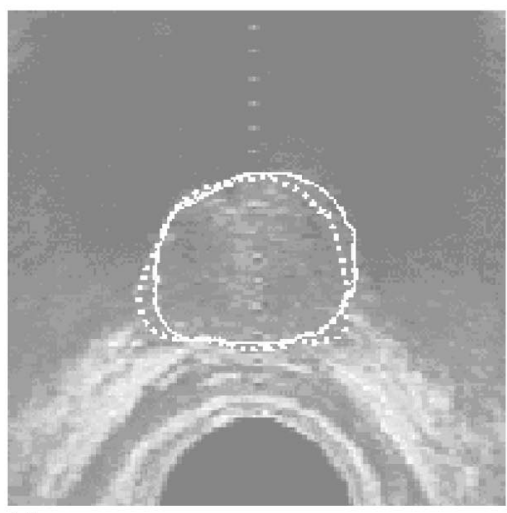

(d)

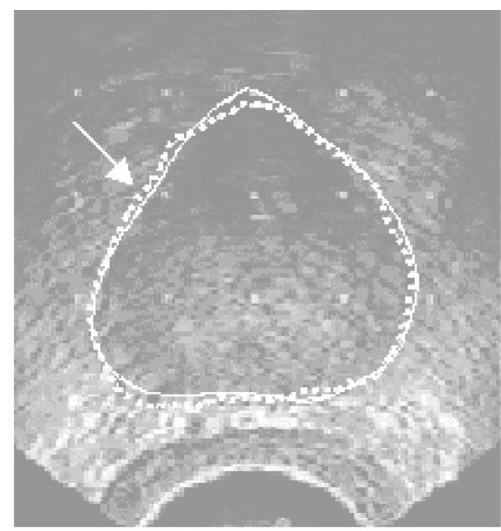

(b)

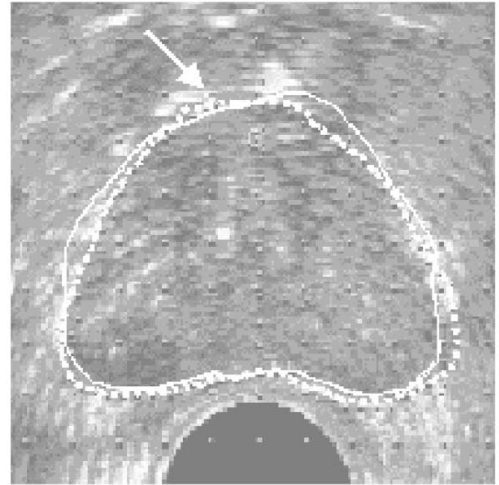

(d)
FIG. 5. (a) A beginning slice of the prostate. (b) The prostate image (using a larger magnification) with the initial contour overlaid (dashed lines). (c) The prostate image with the final contour generated by the model. (d) The prostate image with both the manual contour (solid lines) and the model contour (dashed lines). Note the good agreement between the model and the manual contour.
FIG. 6. Two examples of comparison between the model result with and without using the gradient of the local standard deviation of gray levels $(\nabla \delta)$ in calculating $E_{\mathrm{im}}$. The model results and the manual results are shown using solid and dashed lines, respectively. The images (a),(c) show the results without using $\nabla \delta$ and the images (b),(d) show the results with using $\nabla \delta$. The white arrows point to the areas where significant differences are noticed. 
More studies are needed to confirm the hypothesis that including the standard deviation of the image pixels in calculation of image energies indeed improves the segmentation. Figure 6 shows two examples of comparison between the model result with and without incorporating the local standard deviation of the image gray level $(\nabla \delta)$ in calculating the image energies, $E_{\text {im }}$. Figure 6 shows some improvements in the model results when $\nabla \delta$ is incorporated. In particular, the white arrows in Fig. 6 point to the regions where the effect is most visible. However, more studies are needed to confirm this hypothesis.

Overall, the model generates reproducible results and gives an accuracy of near $89 \%$, and a speed up of up to threeand sixfold compared to a totally manual outlining. Future work on this research can be directed toward including texture features in the calculation of image energies and using landmarks or a priori knowledge for automatic initialization of the model.

\section{ACKNOWLEDGMENTS}

This work was supported in part by NSF Grant BES9911084. The authors are thankful to Alberto de la Zerda and Rabih Hammoud for their help in data acquisition.

a) Author to whom correspondence should be addressed; electronic mail: aghanei@engin.umich.edu

${ }^{1}$ S. D. Pathak, V. Chalana, D. R. Haynor, and Y. Kim, "Edge guided delineation of the prostate in transrectal ultrasound images," Proceedings of the First Joint BMES/EMBS IEEE Conference, 1999, Vol. 2, 1056 pp.

${ }^{2}$ H. M. Ladak, D. B. Downey, D. A. Steinman, and A. Fenster, "Semiautomatic technique for segmentation of the prostate from 2D ultrasound images," Proceedings of the First Joint BMES/EMBS Conference 1999 IEEE, Engineering in Medicine and Biology 21st Annual Conference, Vol. 2, 1144 pp

${ }^{3}$ C. Knoll, V. Grau, M. Alcaniz, S. Albalat, C. Montserrat, H. U. Lemke,
K. Inamura, and M. W. Vannier, "A new conformal radiotherapy planning system for prostate carcinoma," Proceedings of Computer Assisted Radiology and Surgery, CAR 97, 25-28 June 1997, Berlin, Germany, 1997, pp. 375-380.

${ }^{4}$ C. Knoll, M. Alcaniz, V. Grau, C. Montserrat, and M. C. Juan, "Outlining of the prostate using snakes with shape restrictions based on the wavelet transform," Pattern Recogn. 32, 1767-1781 (1999).

${ }^{5}$ C. Knoll, M. Alcaniz, C. Monserrat, V. Grau, and M. C. Juan, "Multiresolution segmentation of medical images using shape-restricted snakes," Proc. SPIE 32, 1767-1781 (1999).

${ }^{6}$ C. H. Chen, J. Y. Lee, W. H. Yang, C. M. Chang, and Y. N. Sun, "Segmentation and reconstruction of prostate from transrectal ultrasound images," Biomed. Eng. Appl. Basis-Commun. 8, 287-292 (1996).

${ }^{7}$ C. H. Chen, J. D. Lee, and H. B. Hsieh, "3D reconstruction of the prostate from transrectal ultrasound images," Biomed. Eng. Appl. Basis Commun. 11, 1-10 (1999).

${ }^{8}$ W. D. Richard and C. G. Keen, "Automated texture-based segmentation of ultrasound images of the prostate," Comput. Medical Imag. Graphics 20, 131-140 (1996).

${ }^{9}$ J. S. Prater and W. D. Richard, "Segmenting ultrasound images of the prostrate using neural networks," Ultrason. Imaging 14, 159-185 (1992).

${ }^{10}$ K. H. Englmeier, R. Hecker, and S. J. Poppl, "A computer assisted estimate of the volume of the prostate gland obtained from transrectal ultrasonic tomograms," Proceedings of the fifth DAGM Symposium on Pattern Recognition, October 1983, Karlsruhe, Germany, 1983, Vol. 35, pp. 361-366.

${ }^{11}$ A. Ghanei, H. Soltanian-Zadeh, and J. P. Windham, "Segmentation of hippocampus from brain MRI using deformable contours," Comput. Med. Imaging Graphics 22, 203-216 (1998).

${ }^{12}$ A. Ghanei, H. Soltanian-Zadeh, and J. P. Windham, "A 3D deformable surface model for segmentation of objects from volumetric data in medical images," Comput. Biol. Med. 28, 239-253 (1998).

${ }^{13}$ P. H. Todd and R. J. Y. McLeod, "Numerical estimation of the curvature of the surfaces," Comput. Aided Design 18, 33-37 (1985).

${ }^{14} \mathrm{R}$. Rao and J. Ben-Arie, "Optimal edge detection using expansion matching and restoration," IEEE Trans. Pattern Anal. Mach. Intell. 16, 11691182 (1994).

${ }^{15}$ N. R. Czerwinski, L. D. Jones, and D. W. O'Brien, "Edge detection in ultrasound speckle noise," IEEE Int. Conf. Image Process. 3, 304-308 (1994). 